\title{
Turbulent Transition in Plane Couette Flows
}

\author{
H. S. Dou ${ }^{1}$, B. C. Khoo ${ }^{2}$, K. S. Yeo ${ }^{2}$ \\ ${ }^{1}$ Temasek Laboratories, National University of Singapore, Singapore 117508 \\ ${ }^{2}$ Department of Mechanical Engineering, National University of Singapore, Singapore 119260 \\ Email: tsldh@nus.edu.sg; huashudou@yahoo.com
}

\begin{abstract}
In the past, several stability theories have been developed to describe the mechanism of flow instability. These are: (1) The linear stability theory, which can be traced back to Rayleigh (1880) is a widely used method and has been applied to some problems [1]; (2) The energy method (Orr, 1907) based on Orr-Reynolds equations; (3) The weak nonlinear stability theory developcd by Stuart and others; (4) The secondary instability theory proposed by Herbert et al. (1988) and his colleagues. However, there are still significant discrepancies between the predictions obtained using these methods and experimental data; particularly at transition. Dou [2,3] proposed a mechanism with the aim to clarify the phenomenon of transition from laminar flow to turbulence for wall-bounded shear flows. In this mechanism, the whole flow field is treated as an encrgy field. By rigorous derivations based on physics, it is found that that the energy gradient in the transverse direction is able to amplify a velocity disturbance, while the viscous friction loss in the streamwise direction can resist and absorb this disturbance. The flow instability or the transition to turbulence depends on the relative magnitude of these two roles of energy gradient amplification and viscous friction damping of the initial disturbance. The analysis has obtained very good consistent agrecment for the plane Poiseuille flow and the pipe Poiseuille flow. This theory is named as "energy gradient theory". In this theory, the flow instability is characterized by a function $K$ of coordinates and the disturbance amplitude. In this paper, using the cnergy analysis, the equation for calculating $K$ in plane Couette flow is derived. It is demonstrated that the critical value of $K$ at the critical condition which is observed from experiments [4 6] is about 370 for plane Couette flow. This value is about the same as for plane Poiseuille flow and pipe Poiseuille flow (385 389). These results demonstrate that the critical value of $K_{\max }$ for wall-bounded parallel flows including both pressure driven and shear driven flows is about 370 389. This consistency also suggests that the mechanisms of instabilities in wall-bounded parallel shear flow are perhaps the same. They are all dominated by the transverse energy gradient and the streamwise flow cnergy loss. The results obtained in this study provide further basis for better understanding of the mechanism of instability and transition to turbulence in parallel shear flows, and provide a basis for the modelling and prediction of the transition process.
\end{abstract}

\section{REFERENCES}

1. Schmid PJ, Henningson DS. Stability and Transition in Shear Flows. New York: Springer-Verlag, 2000

2. Dou HS. Mechanism of flow instability and transition to turbulence. International Journal of Non-Linear Mechanics, 2006;41:512-517

3. Dou HS. The physics of flow instability and turbulent transition in shear flows, Technical Report of National University of Singapore, 2006. http://arxiv.org/abs/physics/0607004

4. Tillmark N, Alfredsson PH. Experiments on transition in plane Couette flow. J. Fluid Mech., 1992;235:89-102

5. Daviaud F, Hegseth J, Bergeé P. Subcritical transition to turbulence in plane Couette flow. Phys. Rev. Lett., 1992;69:2511-2514

6. Malerud S, Malfy KJ, Goldburg WI. Measurements of turbulent velocity fluctuations in a planar Couette cell. Phys. Fluids, 1995; 7:1949-1955 